\title{
Discovery of the Major Mechanism of Global Warming and Climate Change
}

\author{
Paul C. Rivera ${ }^{*, 1}$ and Tariq Masood Ali Khan ${ }^{2}$ \\ ${ }^{1}$ Hymetocean Peers Co. Antipolo City, Philippines \\ ${ }^{2}$ Institute of Environmental Studies, University of Karachi, Pakistan
}

\begin{abstract}
Statistical analysis of the number of destructive earthquakes versus global temperature and greenhouse gases revealed very significant correlations. The motion of the North Pole, deduced from the geomagnetic polar shift data, is highly correlated with major earthquakes. This is an indication that the frequent occurrence of major earthquakes had increased earth's obliquity and induced global warming and possibly emission of greenhouse gases. It was shown by a simple model developed here that seismic-induced oceanic force could enhance the obliquity leading to increased solar radiative flux on earth. The increase of the absorbed solar radiation due to polar tilt was also confirmed by SOLRAD model which computed a net gain of solar radiative forcing due to enhanced obliquity. SOLRAD also revealed a poleward gain of solar radiative flux which could have facilitated the observed polar amplification of global warming. Multiple regression analysis also showed that polar shift and solar irradiance played a major role in the temperature rise and $\mathrm{CO}_{2}$ increase in recent years. The analysis showed that obliquity change due to North Pole shift and total solar irradiance accounted for $63.5 \%$ and $36.4 \%$ respectively, while $\mathrm{CO}_{2}$ changes accounted for $0.1 \%$ of the observed global warming. Preliminary simulations conducted with EdGCM climate model also showed that enhanced obliquity increases the absorbed solar radiative flux, surface air and ocean temperatures, and decreases ocean ice cover. This study confirmed in several ways that earthquake-perturbed obliquity change, and not greenhouse effect, is the major mechanism governing the present global warming and climate change problem.
\end{abstract}

Keywords: Major earthquakes, polar shift, obliquity change, global warming, climate change, SOLRAD model, EdGCM.

\section{INTRODUCTION}

Many disasters presently occurring on earth are climate-related. Few people realize that earthquakes, and their potential impact on the rotation of the earth on its axis, have something to do with the global warming and climate change problem. Early in the 19th century [1], studied the possible impact of earth's tilt (i.e. obliquity) on the global climate and found that several 'thermal days' would result when the earth is tilted further and this would cause the global temperature to rise with associated changes in sea level and vegetation near the poles. At present, rise in greenhouse gas (GHG) emissions especially carbon dioxide $\left(\mathrm{CO}_{2}\right)$ due to anthropogenic sources had been blamed as the major factor triggering the global warming and climate change problem [2]. However, the anthropogenic input of $\mathrm{CO}_{2}$ is a mere $3 \%$ of the global load as compared to the $97 \%$ input of natural sources emanating from the permafrost regions, oceans and the continents [3]. In addition, the observed increase of $\mathrm{CO}_{2}$ concentrations from anthropogenic and natural sources cannot explain the increasing number of destructive earthquakes and volcanic eruptions

*Address corresponding to this author at the Hymetocean Peers Co. Antipolo City, Philippines; Tel: +632-646573; Fax: +632-6465730;

E-mails: paulcrivera2@yahoo.com, pcrivera@gmail.com presently occurring in many regions on earth. Recent studies also found that global warming could be the cause of the increasing $\mathrm{CO}_{2}$ concentrations due to increasing respiration flux of surface soils as temperature rises [4]. Using Global Climate Models, it was shown that natural forcing due to volcanic activity and solar radiation cannot account for the observed global warming after 1970 [5]. It was pointed out that the observed warming during the last part of the $20^{\text {th }}$ century could not be explained by natural sources alone and that the combined influence of greenhouse gases and natural factors including volcanics, solar radiation, and sulfate aerosols could explain the observed warming trend. However, the possible change of the earth's tilt on its axis (i.e. short-term obliquity change), as a natural cause of global warming was neglected in the global climate simulations. A major part of the problem can be traced from the accepted 41,000 -year periodicity of the obliquity as previously established under the Milankovitch astronomical theory of climate change. The cyclical change of obliquity is thus too long and global climate models do not assume short-term annual variability and generally consider a constant obliquity.

It is a long-established fact that the obliquity of the earth causes the seasons to change leading to shortterm seasonal variability of the climate. The obliquity of the planet earth had been estimated in the early $20^{\text {th }}$ 
century by Milankovitch to vary from $22.1-24.5^{\circ}$ and that the present trend is a declining phase from the average tilt of $23.44^{\circ}$. However, more accurate astrophysical modeling conducted in [6] suggested that the earth's obliquity ranges from $19-28.4^{\circ}$ from the ecliptic. The new planetary model is based on the Hermite Integrator with Individual Time-step Scheme (HITS), a numerically more accurate method as compared with the conventional method used in the early $20^{\text {th }}$ century by Milankovitch. It was pointed out that the moon, sun and the planets of the solar system can affect the obliquity of the earth through external torques. Laskar et al. found that the moon can regulate the global climate by limiting the value of the earth's obliquity [7]. It is possible however, that the obliquity of the planet earth can change in the short-term as a result of the dynamic variation of oceanic pressure within the planet itself. The Chandler wobble, which puzzled scientists for a century, proves that the polar motion and small planetary tilt can change almost every year (i.e. once every 1.2 years) due to physical changes in the oceans. NASA confirmed that changes in the earth's obliquity due to the Chandler wobble is largely due to the changing pressure and salinity distribution at the bottom of the oceans (www.jpl.nasa.gov) [8]. It is therefore possible that earthquake-induced tsunamis and the associated strong pressure and force in the oceans could also alter the planetary obliquity. A major earthquake (e.g. the December 2004 temblor off western Sumatra) could impart a very strong pressure in the ocean bottom and trigger a series of destructive tsunami waves [9]. The seismic-generated oceanic pressure, torque and force due to the great earthquake could change the planetary obliquity by altering the lunar force of attraction on earth.

The main objective of this study is to determine the major cause of the global warming and climate change problem and to determine the relative contributions of the climate predictors using available observations. The impact of forced axial tilting of the earth and the possible cause of the enhanced obliquity are considered. A new theoretical model of obliquity change is presented in this paper. Available observations of major earthquakes and motion of the geomagnetic poles are used to determine the change in the earth's obliquity. To prove the enhanced planetary obliquity, a simple measurement technique is proposed. Coupled with observed changes in solar irradiance, time-series analysis of the dataset is performed using multiple regression to determine the relative contributions of the major factors affecting the global climate. The impact of the enhanced obliquity on the global climate was also simulated using the EdGCM global climate model (www.edgcm.columbia.edu) [10]. The model results are compared to available observations to determine if the assumed natural forcing could indeed be the major cause of the temperature rise and abrupt climate change problem presently occurring on earth.

\section{METHODS}

\subsection{Data Sources and Statistical Analysis}

A simple measurement technique is proposed in this study to determine the enhanced obliquity of the planet. To determine the effect of enhanced obliquity, a theoretical solar radiation model (SOLRAD) was also applied. The model was run with different obliquity values to determine the associated change in the radiative flux received by the earth. Preliminary climate simulations using the Education Global Climate Model (EdGCM) were also conducted to determine the impact of enhanced obliquity on the major climate parameters particularly surface air temperature. The results are compared with available observations.

Data on the number of major earthquakes were obtained from the public domain (www.en.wikipedia.org/wiki) [11, 12]. On the other hand, data on global temperature, $\mathrm{CO}_{2}$ and other GHG concentrations, and Total Solar Irradiance (TSI) were obtained from the National Climate Data Center (NCDC) of the National Oceanic and Atmospheric Administration (www.ncdc.noaa.gov) [13]. The motion of the geomagnetic North Pole and estimated obliquity was obtained from the National Geophysical Data Center (NGDC) of NOAA (www.ngdc.noaa.gov) [14]. Statistical analyses such as linear correlation and multiple regression were used on the various climate parameters. Strength of relationships between the various climate parameters was deduced from the resulting correlation coefficients and standard errors. The absolute contributions (i.e. attribution) of the major climate factors on global warming were determined using multiple regression.

\section{RESULTS AND DISCUSSION}

\subsection{The Role of Major Earthquakes on Obliquity Change and Polar Shift}

Time-series analysis of earthquake count revealed that the number of destructive earthquakes showed an 
abrupt rise before the turn of the millenium. In addition, the geomagnetic polar shift data showed a possible increase of the planetary obliquity which coincided with the increase of major earthquakes. The data on the motion of the geomagnetic poles had been derived from ship log data since 1590 and recorded by the National Geophysical Data Center (NGDC) of the National Oceanic and Atmospheric Administration (NOAA). The observed shift of the geomagnetic North Pole since 1880 is indicative that the geographic North Pole may have actually shifted southwards. Because of the suddenness of the polar shift (i.e. $14^{\circ}$ from 1900 2010), the proposed northward motion of the geomagnetic North Pole from Arctic Canada towards Russia might actually indicate that the geographic North Pole has shifted southwards. Thus, the obliquity might have increased and reversed the Milankovitchpredicted trend. The planetary obliquity from the conventional Milankovitch theory should show a very gradual decreasing trend from the present mean of about $23.45^{\circ}$. The polar shift data, however, showed a very abrupt change in the location of the geomagnetic North Pole. From the polar shift data, the obliquity change was estimated from the annual and decadal changes of the recorded latitude position of the geomagnetic North Pole. The obliquity change which was computed from the decadal change of the latitudinal position of the magnetic North Pole and the number of major earthquakes $(\boldsymbol{E C})$ are shown together in Figure 1 (left). The result revealed that the obliquity change started to increase dramatically since 1970 . This obliquity change is linearly related to the number of major earthquakes plus a constant (Figure 1, right). As the data showed, the recorded number of major earthquakes increased abruptly during the same period. Since 1995, the increase in the obliquity change is very evident and so is the number of major earthquakes. It should be noted that an 'internal' torque

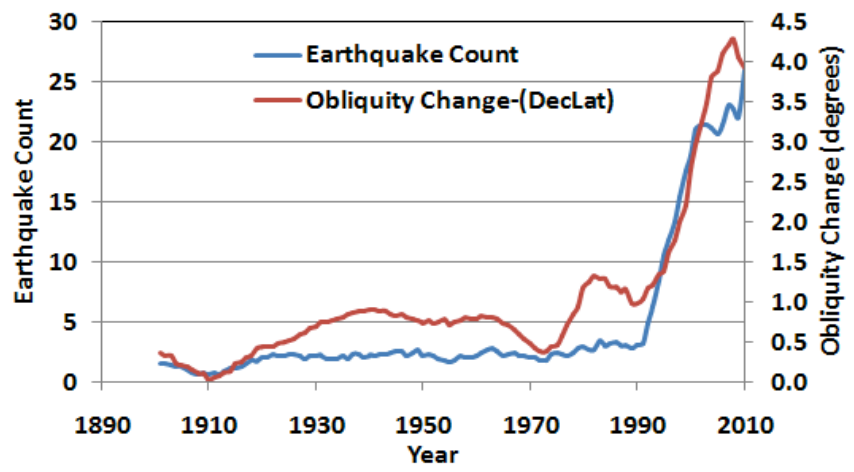

is exerted during an earthquake within the lithosphere and the hydrosphere including the oceans (i.e. the rise in tsunami occurrence since 1990 is obvious). Therefore, major earthquakes could perturb the earth's axis and might have caused the sudden polar motion as shown by the polar shift data. With an everincreasing number of destructive earthquakes, the effect of external torques from distant planets on the motion of the earth's axis could be outweighed by internal torques.

Based on the NGDC data, the annual obliquity values were then computed from the sum of the mean obliquity of $23.4^{\circ}$ and the decadal change of the latitudinal position of the geomagnetic North Pole. As shown in Figure 2, the estimated obliquity and the latitudinal shift of the geomagnetic North Pole is highly correlated with the number of major earthquakes $\left(r^{2}=\right.$ 0.978 and $\left.r^{2}=0.90\right)$. Chao and $I z$ was the first to conduct a statistical test to correlate the occurrence of earthquake with polar motion but only a weak correlation was found during the period 1977-1991 [15]. With a longer data set used in this study, the relationship between the earth's obliquity $(O B L)$ and the number of destructive earthquakes $(E C)$ was found to be highly correlated (Figure 2, left) and represented by a simple equation of the form:

$O B L=23.4 e^{0.004 E C}$

Remarkably, the obliquity is equal to the presentday value of $23.4^{\circ}$ if there were no major earthquakes (i.e. $E C=0$ ). This implies that a major shift in the geomagnetic North Pole and consequently the geographic North Pole, could have resulted from internal torques during earthquakes. The effect of external torques from other planets on the planetary obliquity has been shown by Milankovitch to vary within a period 41,000 years.



Figure 1: The number of major earthquakes $(E C)$ and obliquity change estimated from the decadal change of the North Pole tilt (NGDC). 

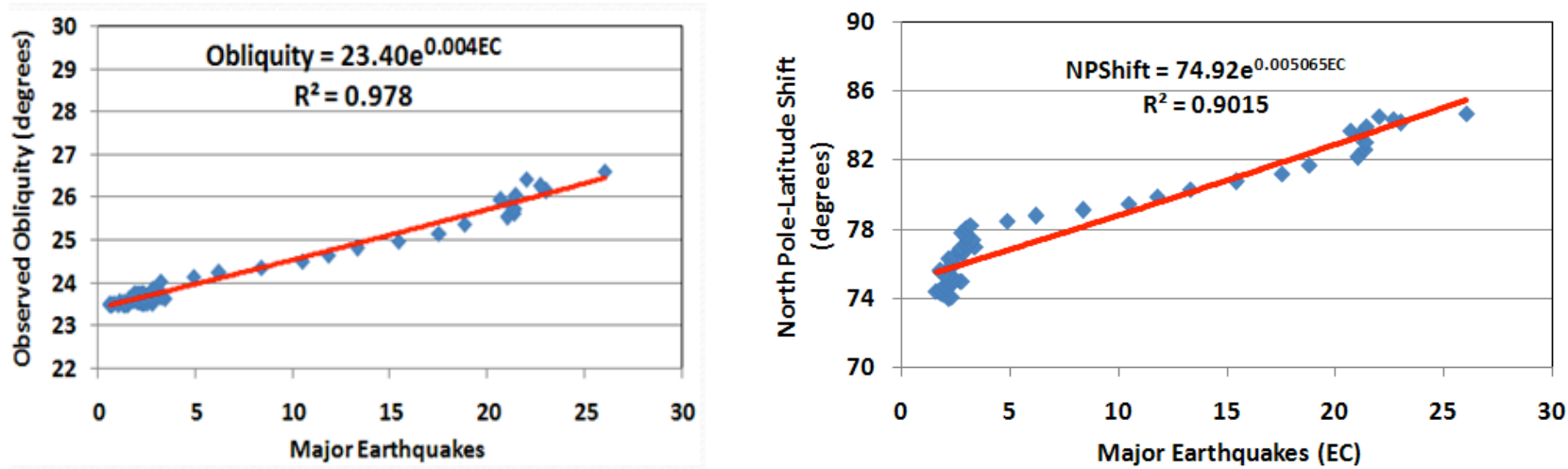

Figure 2: Significant correlations exist between the observed latitudinal change of geomagnetic North Pole, the obliquity and the number of major earthquakes.

Using the 7-year moving average of the North Pole (NP) shift data, it was also found that during the period 1950-2010, the latitudinal change of the geomagnetic north pole was shown to have a very high correlation coefficient $\left(r^{2}=0.90\right)$ with the number of major earthquakes (Figure 2 , right) and is represented by the equation;

$N P=74.92 e^{0.00506 E C}$

where $\boldsymbol{N P}$ is the observed latitudinal shift of the magnetic North Pole in degrees. Similar to the estimated obliquity, the NP shift reverts to an average constant value, if there were no major earthquakes (e.g. $E C=0)$. It is very likely therefore, that the polar motion (geomagnetic and geographic North Pole) was largely driven by major earthquakes. The geographic North Pole has shifted southwards and so the planetary obliquity has increased. The enhanced obliquity of earth is shown below to have a considerable influence on the global climate due to the associated net gain in the solar radiative flux absorbed by the planet.

Since major earthquakes were shown to have triggered the polar motion and obliquity change of the planet, the data do not show any clear periodicity. Major earthquakes were just shown by the data to have occurred more frequently during the second half of the $20^{\text {th }}$ century. The sudden increase in the number of destructive earthquakes must have triggered the abrupt change in the earth's obliquity owing to the altered angular momentum and oceanic torques imparted by earthquakes and tsunamis on the predominantly fluid earth, leading to a reversal of the Milankovitch-trend in the planetary obliquity.

\subsection{New Earthquake-Perturbed Obliquity Change (EPOCH) Model}

Earthquakes produce a counteracting force against the earth's normal rotation which is manifested in certain ways including the generation of trans-oceanic mega-tsunamis and the release of tremendous pressure at the seabed. Using a new tsunami generation model $[9,16]$, a very strong oceanic force and associated torque or angular momentum was calculated from the giant submarine quake that generated the Asian Tsunami of December 26, 2004 in the Indian Ocean. The strong pressure from the submarine quake generated a horizontal water current motion whose strength was dictated by the earthquake moment magnitude and focal depth, the oceanic buoyancy frequency, and the slope of the ruptured seabed across the fault line (Figure 3 ). The seismicinduced total oceanic force is equivalent to the sum of the hydrostatic force and the earthquake-induced lateral collision force. The newly proposed tsunami generation mechanism was corroborated and concluded that the great tsunami was largely generated by a strong lateral collision force of the continental slope and the ocean column as shown by independent evidence from seismographs, satellite radar altimeters, and tide gauges in the region [17, 18]. The series of tsunami waves that were generated initially rushed westward and subsequently encircled the globe through the major oceans. This created an imbalance on the existing lunar force of attraction on earth and acted to tilt the earth further, reversing the Milankovitch-predicted decreasing obliquity trend.

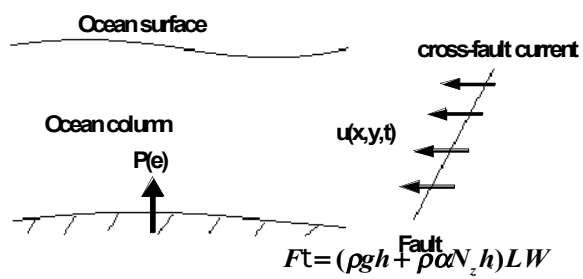

Figure 3: A strong westward oceanic force during major earthquakes counters the normal rotation of the earth. The seismic-induced total oceanic force is equivalent to the sum of the hydrostatic force and the lateral collision force that triggers a mega-tsunami $[9,16]$. 


\subsection{Computed Obliquity Change Due to Earthquake-Induced Oceanic Force}

The great Asian earthquake and tsunami of December 2004 occurred during a period of earth's maximum tilt from the sun and towards the moon (i.e. December 26, 2004 was a full moon). Since the earth's radius is very small $(1.67 \%)$ compared with the earthmoon distance, a balance of forces exists as shown in Figure 4. The earth and its moon, though spherical in shape, are small dots in the universe but are magnified here to show what happens to the earth's axis with a strong lateral oceanic force and associated oceanic torque produced by a major earthquake.

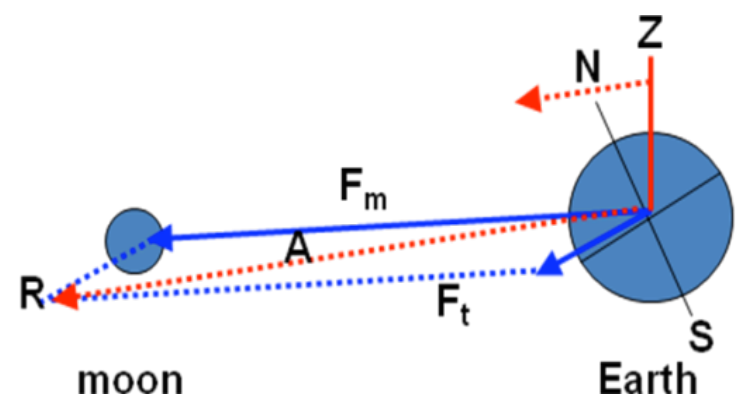

Figure 4: Earthquake-Perturbed Obliquity $\mathrm{CHange}(\mathrm{EPOCH})$ Model showing the altered gravitational pull of the moon on earth due to strong counteracting force (and internal torque) during major earthquakes and tsunamis.

Using Newton's law of gravitation, the gravitational force exerted by the moon with mass $m$, on the earth with mass $M$, separated by a distance $r$ is given by $F_{m}=G \frac{M m}{r^{2}}$. This is equivalent to about $2.0 \times 10^{20} \mathrm{~N}$ and is normally directed towards the moon. The earth has an equivalent gravitational force which could also 'pull' the moon and affect its orbit around the earth. Humlum et al. recently found that natural cyclic variation of the moon's orbit around the earth affects the global climate [19]. The quake-induced tsunami near the equator off Sumatra generated a very strong lateral force that countered the earth's rotation. The induced oceanic torque (clockwise when viewed from the north pole) countered the regular rotation of the earth from west to east, leading to the increased axial tilt or obliquity of the planet. This is one major means whereby an 'internal' torque from the earth itself could alter the planetary obliquity.

The magnitude of the total seismic-induced oceanic force during the giant tsunami is equivalent to the product of the displaced water mass along the ruptured fault and the total oceanic acceleration due to gravitational acceleration and the seismic-induced ocean acceleration. The earthquake-generated oceanic acceleration can be determined from the currentinduced tsunami generation model $[9,16]$ and is strongly dependent on the slope of the perturbed seabed. Off the island of Sumatra, the abrupt increase of water depths towards the west and southwest was responsible for the strong collision force between the continental slope and the oceanic water mass that triggered the tsunami. The total oceanic force can be calculated using the $\alpha$-parameter introduced in [9], namely;

$$
F_{t}=\rho L W\left[g h+\frac{1}{4}\left(\frac{10^{M_{w}} g}{\rho(D+h) v}\right)^{1 / 3} N_{z} \frac{\Delta h}{\Delta s}\right]
$$

Here, $\rho$ is the ocean water density $\left(\mathrm{kg} / \mathrm{m}^{3}\right), g$ is gravitational acceleration $\left(\mathrm{m} / \mathrm{s}^{2}\right), h$ is the water depth $(\mathrm{m})$ where the ruptured fault occurred, $M_{w}$ is the moment magnitude of the earthquake, $D$ is the focal depth $(\mathrm{m}), \quad U$ is the kinematic viscosity of seawater $\left(\mathrm{m}^{2} / \mathrm{s}\right), N_{z}$ is the ocean buoyancy frequency $\left(\mathrm{s}^{-1}\right), \Delta h / \Delta \mathrm{s}$ is the seabed slope or difference of water depth $(\mathrm{m})$ normal to the ruptured fault, $L$ is the length of ruptured fault $(\mathrm{m})$, and $W$ is the width of the fault $(\mathrm{m})$. Using the moment magnitude of the quake $\left(M_{w}=9.3\right)$, focal depth $(14 \mathrm{~km})$ and water depth $(4 \mathrm{~km})$, the oceanic force $F_{t}$ was determined to be about $3.137 \times 10^{19} \mathrm{~N}$. The strong pressure that acted in a large area in the Indian Ocean is proportional to the length of the ruptured faultline times its width (i.e. $1200 \mathrm{~km} \times 550 \mathrm{~km}$ ).

Using the law of cosine in Figure 4, the perturbed lunar force represented by the resultant force $\boldsymbol{R}$ was estimated to be about $2.162 \times 10^{20} \mathrm{~N}$. The oblique angle opposite of $\boldsymbol{R}\left(=156.55^{\circ}\right)$ is the difference between $180^{\circ}$ and the initial obliquity of earth $\left(23.45^{\circ}\right)$. The angle of tilt $A$ was then computed from the law of sine;

$$
A=\sin ^{-1}\left(\frac{F_{t} \sin \left(156.55^{\circ}\right)}{R}\right)
$$

Plugging the values obtained above, it can be shown that the strong westward oceanic force and associated torque generated a small rotational displacement of $\boldsymbol{Z}$ and consequently of the original $\mathbf{N}-\mathbf{S}$ axis of the earth. The tilt of the earth has been increased by $A=3.31^{\circ}$ due to the giant quake and tsunami of December 2004. It should be noted that another strong quake with a magnitude of about $M_{w}=$ 7.1 occurred in the same place about 3 hours later. Assuming that the area affected by the strong pressure 
at the ocean bottom is smaller than the previous one $(600 \mathrm{~km} \times 150 \mathrm{~km})$, an additional tilt of the earth's axis of about $0.421^{\circ}$ would result. This leads to a total increase in the planetary obliquity of about $3.73^{\circ}$. Hence, the earth's obliquity could have been tilted further to about $27.18^{\circ}$ due to the giant Sumatra earthquake and tsunami of December 2004. It is remarkable that the estimated obliquity change of $3.73^{\circ}$ is very close to the observed obliquity change of $3.88^{\circ}$ from the NGDC polar shift data after the 2004 earthquake and tsunami. The lower obliquity change computed above could be due to the exclusion of earthquake-perturbed motion within the lithospere. In addition, the disturbed oceanic area could be underestimated leading to a lesser oceanic mass that was forced into motion during the mega-tsunami. The occurrence of other earthquakes and tsunamis right after the great tsunami of Dec. 2004 could also account for the higher obliquity change (i.e. $4.27^{\circ}$ in 2008) given by the polar shift data.

It should be noted that the oceanic angular momentum generated by the magnitude 9.3 earthquake of December 2004 within the ruptured fault amounted to about $4.46 \times 10^{27} \mathrm{~kg}-\mathrm{m}^{2} / \mathrm{s}$, which is an order of magnitude (about 35 times) greater than the global atmospheric angular momentum (AAM). This indicates that major earthquakes and tsunamis can have a considerable impact on the motion of the predominantly fluid-covered planet and are capable of enhancing the planetary obliquity by overall movement within the oceans alone (exluding perturbations in the hydrosphere, lithosphere, and atmosphere). As shown by the observed polar motion and the newly estimated obliquity (below), it is possible that major earthquakes could have started to tilt the planet even before the end of the $20^{\text {th }}$ century. The enhanced tilt, which is within the newly simulated earth's obliquity [6], cannot be easily restored by the gravitational force of the moon and distant planets leaving the earth with enhanced obliquity that reversed the Milankovitch-predicted decreasing trend.

The newly proposed seismic-generated obliquity change and overall motion of the North Pole can also have significant impact on the occurrence of El Niño Southern Oscillation (ENSO) and the Pacific Decadal Oscillation (PDO). The polar shift data showed significant obliquity change that occurred every decade. The decadal change was shown in this study to have significant correlation with global temperature rise. Thus, this could be the major cause of the PDO and ENSO events. Kosek et al. showed a significant correlation between the polar motion and the occurrence of ENSO during the period 1982-83 and 1997-1998 [20].

\subsection{Observed Planetary Obliquity at Sunrise and Sunset}

Due to the obliquity of the earth on its axis and the rotation of the planet around the sun, the declination angle of the sun varies seasonally. If the earth were not tilted on its axis (i.e. obliquity $=0^{\circ}$ ), the declination would be $0^{\circ}$. The earth is initially tilted by about $23.45^{\circ}$, and the declination angle of the sun originally spans about $46.9^{\circ}$ in the horizon throughout the year.

To determine if the earth's obliquity has indeed increased, a simple measurement technique was conducted. An observer can use a magnetic compass and a straight rod for the simple experiment which can be done before sunset or at sunrise. By fixing the rod vertically upwards and measuring the angle that the shadow of the rod makes on the compass (i.e. make sure that the compass arrow points towards the north), one can determine the value of the enhanced obliquity of the planet. The obliquity and declination angle should attain a maximum negative value of about $23.45^{\circ}$ on December 21 as the maximum declination of the sun during the winter solstice is about $-23.45^{\circ}$. The obliquity is then found by subtracting $90^{\circ}$ before sunset (or $270^{\circ}$ at sunrise) from the observed angle in the compass. If the resulting angle is about $-23.45^{\circ}$ during the winter solstice, this implies that the earth has not been tilted further. However, if the resulting angle is less than $-23.45^{\circ}$, this proves that the earth has tilted further.

Using a magnetic compass, the simple experiment was conducted before sunset during the period 14-17 December 2010 and the observed angle was about $62.5^{\circ}$ giving an obliquity of about $-27.5^{\circ}$. Similar experiment was also conducted at sunrise during the winter solstice of December 21, 2010. The observed obliquity angle was about $-27.5^{\circ}$. Another experiment was conducted before sunset during the summer solstice of June 2011. The observed angle was about 117.5 giving an obliquity of about $27.5^{\circ}$ (e.g. $117.5^{\circ}$ $90^{\circ}$ ). The same experiment was conducted at sunset in December 20 and December 31, 2011 and the observed obliquity angle was about $-27.5^{\circ}$. Due to the curvature of the earth, the actual obliquity could be slightly smaller since measurement was done when the sun is slightly above the horizon (i.e. the horizon was 
partly covered with clouds at 0600 and 1800 hours). It should be noted that the estimated obliquity from the NGDC data set in the year 2010 is about $27.37^{\circ}$. Thus, the obliquity is no longer on a gradual declining phase but has increased from the mean obliquity of $23.45^{\circ}$. The Milankovitch-trend of obliquity is thus reversed. The observed and computed departure of the planetary obliquity from its mean value of $23.45^{\circ}$ can have a profound effect on the safety of aviation and navigation.

\subsection{Enhanced Obliquity and Altered Global Solar Radiation}

Changes in the planetary obliquity have a considerable impact on the global climate by altering the absorbed global solar radiation. As shown by the observed and computed values above, the obliquity of the earth could be enhanced during the occurrence of major earthquakes and tsunamis. This could affect the earth's climate in several ways including an increase in the radiative flux received by the earth and associated surface heating and potential release of greenhouse gases. To further confirm this hypothesis, a theoretical solar radiation model (SOLRAD) with altered obliquity values was run.

SOLRAD (version 1.2) which was written by Pelletier is a simplified solar radiation model that is based on clear sky conditions and therefore does not take into account the dynamics of cloud formation and other complex atmospheric processes [21]. The solar position calculations in this Excel/VBA application are a translation of NOAA's solar position calculator. Solar radiation calculations were based on the models [2224]. For convenience, only the results of the Bird and Hulstrom model are presented and discussed. The potential change in the global solar radiation due to enhanced obliquity as computed with this model was almost similar with the other models. The computed

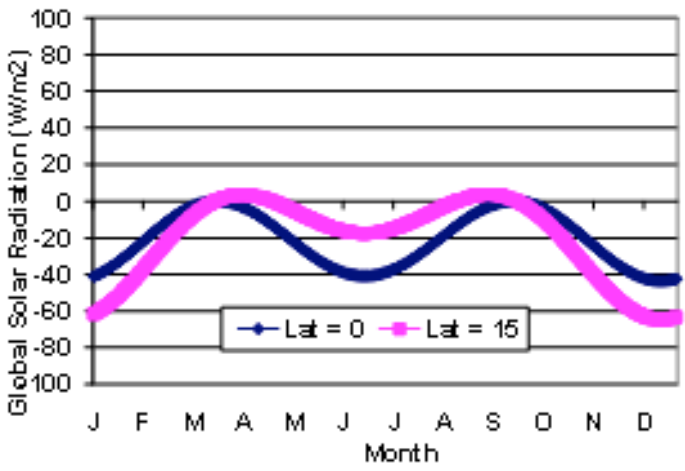

solar radiative flux with an obliquity value of $23.4^{\circ}$ was subtracted from computed radiative flux with enhanced obliquity of $26.15^{\circ}$ and $28.4^{\circ}$. For the later, the computed changes for each latitude circles are shown in Figure 5. It can be seen that the monthly change is all negative at the equator, whereas positive and negative changes representing gain and losses in radiative fluxes occur at higher latitudes. At latitude 30$45^{\circ} \mathrm{N}$, the reduction of solar radiation due to enhanced obliquity ranges from $-60 \mathrm{~W} / \mathrm{m}^{2}$ to $-80 \mathrm{~W} / \mathrm{m}^{2}$ from November to February which could easily explain the occurrence of (early and late) extreme winters nowadays. Above the Arctic circle (and also at the Antarctic circle), the gain in radiative flux is so high that the loss is so small in comparison. This could explain the accelerated melting of polar ice sheets and exposure of permafrost in recent years.

When the daily radiative fluxes are averaged over a year, the existence and major cause of global warming becomes more evident. The computed latitudinal variation of the annual global solar radiation and the net changes of the solar radiative flux with enhanced obliquities (on a horizontal surface on earth with clear sky conditions) are shown in Figure 6 . The result showed a positive gain in solar radiative flux beyond $45^{\circ}$ and $+45^{\circ}$. When these were averaged from North to South Poles, a positive gain in the solar radiative flux amounting to over $4 \mathrm{~W} / \mathrm{m}^{2}$ was obtained with enhanced obliquity of $27^{\circ}$. This is the same amount of absorbed radiation being attributed to the decrease of Outgoing Long-wave Radiation (OLR) assumed to be trapped by increasing greenhouse gases [2]. Assuming that the total cloud cover is about $67 \%$, this would result to a net gain in the solar radiative flux of about $2.8 \mathrm{~W} / \mathrm{m}^{2}$ absorbed by the earth. It should be noted that the estimated solar radiative flux here is equivalent to the infrared radiative (IR) climate forcing being attributed to

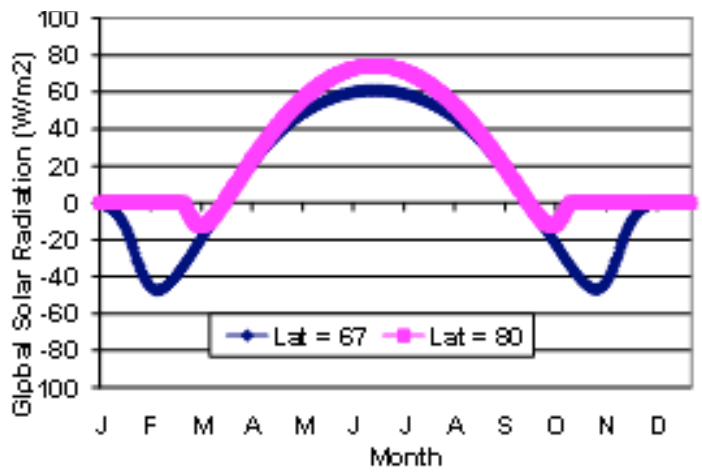

Figure 5: Change in the monthly radiative flux $\left(\mathrm{W} / \mathrm{m}^{2}\right)$ received by the earth due to enhanced obliquity. The altered solar radiation absorbed by the planet could be the real cause of the present global warming and climate change problem. 

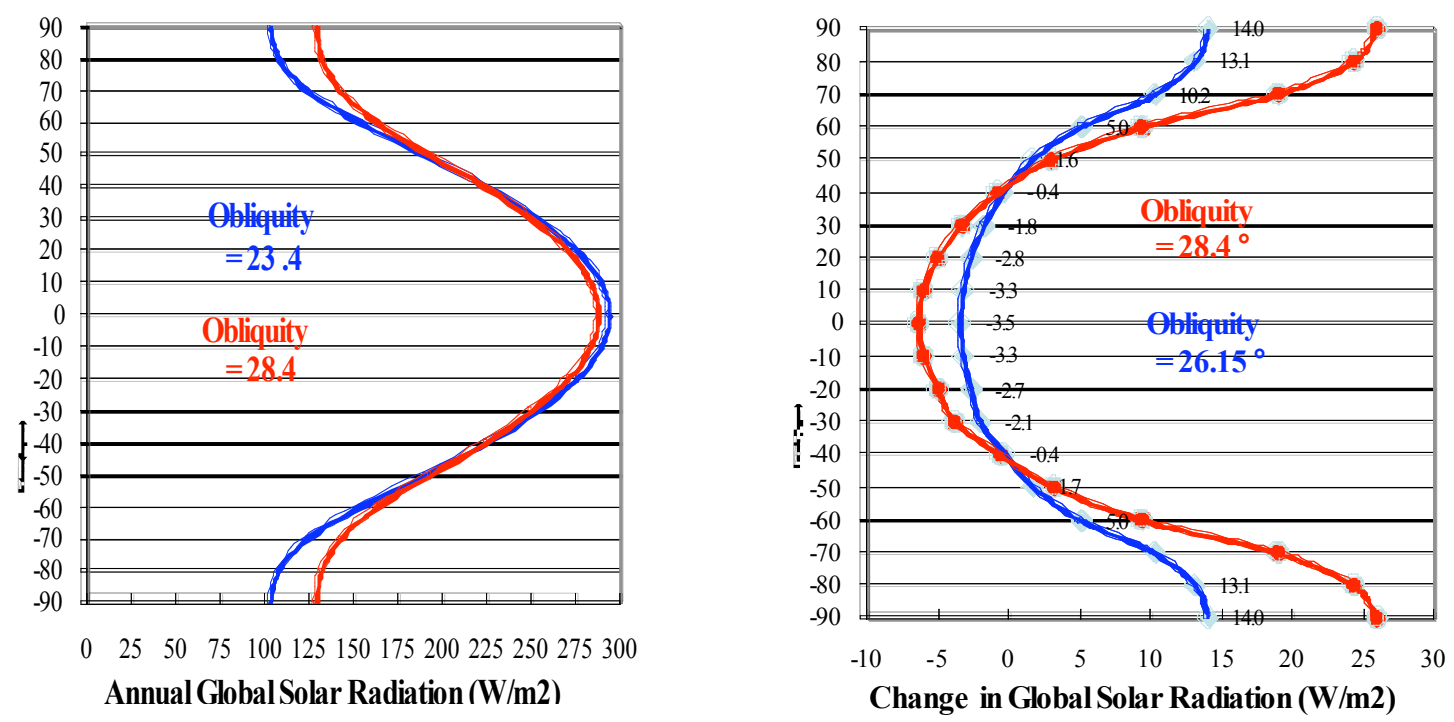

Figure 6: Computed annual average and latitudinal change of global solar radiation $\left(\mathrm{W} / \mathrm{m}^{2}\right)$ on a horizontal surface on earth under clear sky. The high radiative flux with enhanced obliquity near the poles causes the observed polar amplification of global warming.

greenhouse gases. The enhanced obliquity and associated changes of solar radiative flux on earth could therefore be the real cause of accelerated melting of polar ice caps and glaciers in recent years and not greenhouse effect, since the earth's poles now experience more direct radiation from the blazing sun (which also consists of a strong infrared component). The infrared radiation reflected by the earth and trapped by greenhouse gases is quite small in terms of energy magnitude as compared to the energy of the infared solar radiation.

The obliquity of the earth could have been enhanced by the giant Sumatra quake and tsunami to about $27^{\circ}$ and the present obliquity as estimated by SOLRAD is about $27.67^{\circ}$. This implies that other major earthquakes could be responsible for the additional tilt of the earth's axis. With the frequent occurrence of major earthquakes at present, the obliquity of the planet could be very close to the maximum obliquity of $28.4^{\circ}$ as predicted [6].

\subsection{Climate Change Attribution using Multiple Regression}

For a more comprehensive climate change attribution, data on total solar irradiance were incorporated in this study. The TSI data set was taken from Steinhilber et al. as compiled by NCDC-NOAA [25]. These were derived using open solar magnetic field and extended during the holocene period. In this study, only the recent data set starting from 1880 were used. Since the data set was originally presented every 5 years, simple averaging was implemented to interpolate the yearly TSI data. The annual TSI data are plotted with the original TSI data (in red squares) in Figure 7.

The data on total solar irradiance showed a gradual and oscillatory increase of solar radiation from 19011994. During this period, there was a gradual increase from about $1365.5 \mathrm{~W} / \mathrm{m}^{2}$ to about $1366.01 \mathrm{~W} / \mathrm{m}^{2}$ in 1993. The solar irradiance suddenly decreased during the years from 1960-1974 but began to increase again thereafter until it reached the maximum value. Since 1995, the irradiance data showed a gradual decrease until the present time with a minimum value that is a bit lower than the minimum in 1973.

A solar radiation tide appeared from the solar irradiance data since 1900 with a noticeable inequality between the two maximum values and the two minimum values. The first peak occurred in 1958 and the second peak appeared in 1988 when global warming was first declared. There appeared a solar periodicity of about 33 years from the new TSI data which is not related to the well-known 11-year sunspot cycle. A third peak, which could be higher than the second, could occur in the future (ca. 2021). It is quite fortunate that the maximum solar irradiance did not coincide with the increase in planetary obliquity. If this happened, the resulting increase of solar radiative flux absorbed by the earth could have been much higher leading to extreme changes in the weather and the 
climate. As the data showed, when the solar irradiance began to decrease in 1994, major earthquakes appeared to have increased in number with the associated increase in planetary obliquity and polar tilt, and dramatic rise of global temperature.

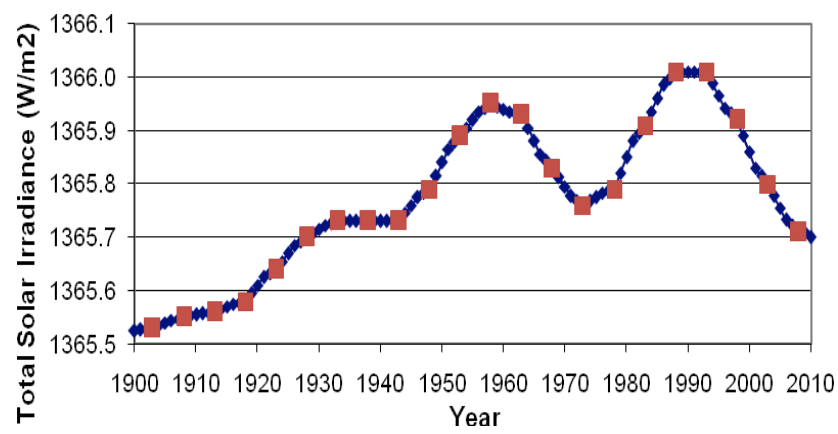

Figure 7: Total solar irradiance from Steinhilber et al. (2009) derived using open solar magnetic field data and averaged annually.

A statistical test that would show the relative contributions of increased obliquity, total solar irradiance and GHG concentrations on the global temperature rise was performed on the data sets using multiple regression in MS Excel. The data set on earthquake count was smoothed by taking the 7-year moving average. All the other data on temperature anomaly and GHG concentrations were not smoothed since they already represent yearly averaged values from monthly observations.

The multiple regression analysis of the observed data revealed a rather strong positive contribution of polar shift and associated enhanced obliquity on the observed increase of global temperature and $\mathrm{CO}_{2}$ concentration [26]. Similar to Fang et al., the regression analysis showed that the annual increment of $\mathrm{CO}_{2}$ concentration is not significantly correlated with global temperature. However, the regression analysis showed

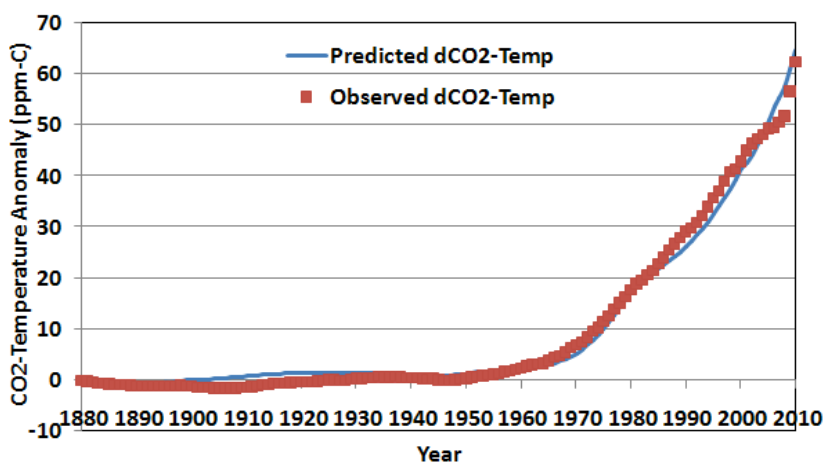

that $\mathrm{CO}_{2}$ and global temperature were largely dictated by obliquity and TSI changes. This shows the possibility that the global warming problem is not dictated by increasing $\mathrm{CO}_{2}$ concentrations but rather by the enhanced obliquity and changes in solar irradiance. Multiplying the $\mathrm{CO}_{2}$ anomaly with the global temperature anomaly and subjecting the product to a 30-year running average revealed a very significant correlation with TSI and obliquity changes as shown in Figure 8 (left). Similar regression analysis of the ratio of $\mathrm{CO}_{2}$ and global temperature versus TSI and North Pole Shift showed a significant correlation with $r^{2}=0.99$ (Figure 8, right).

The impact of obliquity change on the global temperature and $\mathrm{CO}_{2}$ concentrations were also analyzed individually using the observed polar shift data from NGDC since 1880 . The regression analysis in Figure 9 revealed that the rising global temperature and $\mathrm{CO}_{2}$ concentrations are largely dictated by the changes in obliquity and TSI. Both analyses yielded very high correlation $\left(r^{2}=0.97-0.99\right)$ and low standard errors. These showed the big impact of the obliquity change on the global warming problem, and possibly on the rise of greenhouse gases. The correct timing in the changes of both the global temperature and GHG rise together with the obliquity change and TSI variation was not coincidental. It should be noted that TSI started to decrease after attaining its peak in 1990, but the North Pole shift (and planetary obliquity) was abruptly enhanced after that period possibly due to increased number of major earthquakes, resulting to an accelerated warming since then. The regression analyses also yielded very low standard errors. This proves that variations in the TSI and North Pole shift (or obliquity change) are the major cause of the observed global temperature including $\mathrm{CO}_{2}$ rise. The individual analysis clearly showed that the observed

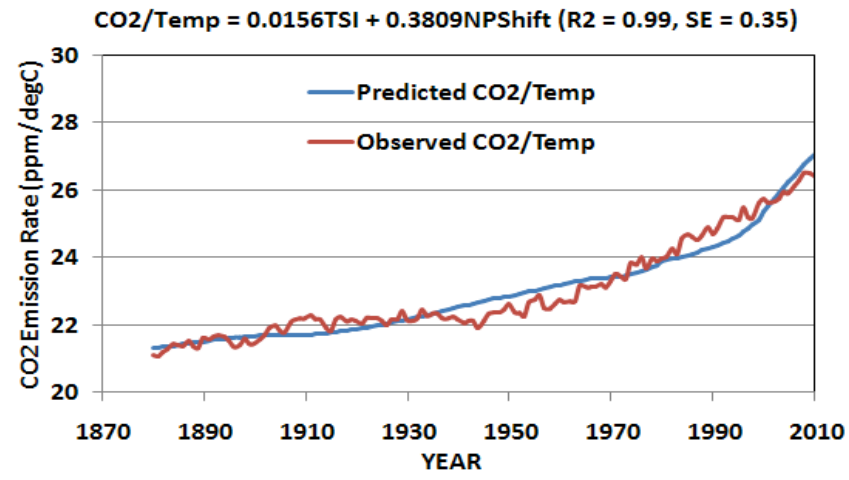

Figure 8: Multiple regression analysis for $\mathrm{CO}_{2}$-temperature anomaly (left) and ratio of $\mathrm{CO}_{2}$ and global temperature (right) versus TSI and smoothed obliquity anomalies. 

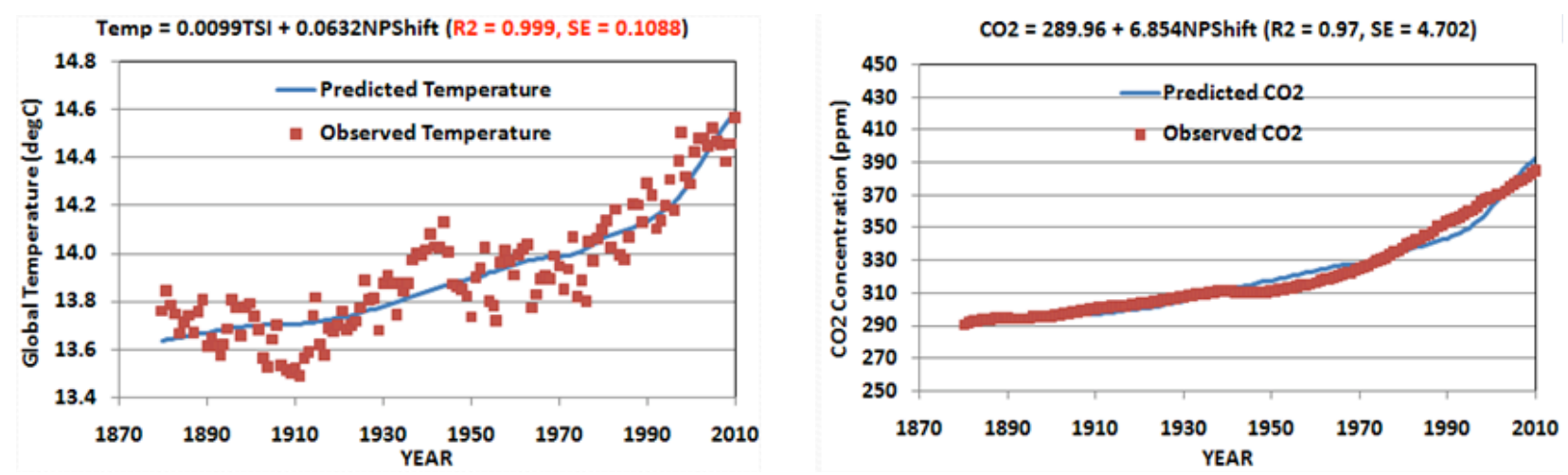

Figure 9: Individual regression analysis for global temperature and $\mathrm{CO}_{2}$ versus Polar Shift and Total Solar Irradiance. $\mathrm{CO}_{2}$ increase is significantly correlated with polar shift data (right).

rise of $\mathrm{CO}_{2}$ concentrations is due mainly to the tilting motion of the north pole (Figure 9, right). Since $\mathrm{CO}_{2}$ respiration fluxes also increase due to increasing temperature and solar heating, the result proves that both global warming and the rise in greenhouse gases are directly related to the enhanced planetary tilting and TSI changes.

The individual regression analyses conducted for global temperature versus $\mathrm{CO}_{2}$ concentration (and vice versa) did not show better results with lower $r^{2}$ values and higher standard errors. The regression analysis for global temperature versus $\mathrm{CO}_{2}$ was compared with the analysis made for global temperature versus the annual increment of $\mathrm{CO}_{2}$ in Figure 10. The regression result for global temperature versus $\mathrm{CO}_{2}$ (Figure 10, left) showed a seemingly good correlation with $r^{2}=0.81$ and low standard error of 0.11 . However, the regression analysis for global temperature versus the annual increment of $\mathrm{CO}_{2}$ (Figure 10, right) showed poor statistics with very low correlation $\left(r^{2}=0.52\right)$ and high standard error $(S E=0.17)$. The results imply that

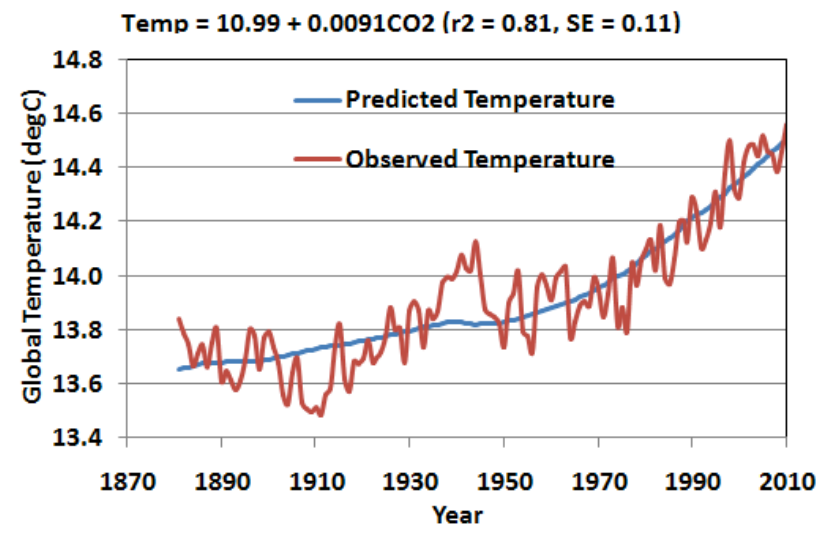

the overall increase in the global temperature cannot be due largely to $\mathrm{CO}_{2}$ increases since the correlation is very low. As concluded by Fang et al. [26], the temperature change has not always been consistent with the change of $\mathrm{CO}_{2}$ concentration, since for several periods the global temperatures decreased or were stable while the atmospheric $\mathrm{CO}_{2}$ concentration continuously increased.

A direct computation of the contributions of $\mathrm{CO}_{2}$, North Pole shift (or obliquity change), and TSI to global warming was done using multiple regression against the observed global temperature variation since 1880 . The analysis made use of the annual increment of $\mathrm{CO}_{2}$, decadal change of the North Pole Shift, and TSI anomaly. The result showed that since the start of the new millenium, the relative contribution of the North Pole shift or planetary tilt outweighed the combined effects of both TSI and $\mathrm{CO}_{2}$ changes (Figure 11, left). The tilt of the North Pole contributed about $63.5 \%$ while TSI change contributed $36.4 \%$ to the observed temperature rise. The annual increment of $\mathrm{CO}_{2}$ showed

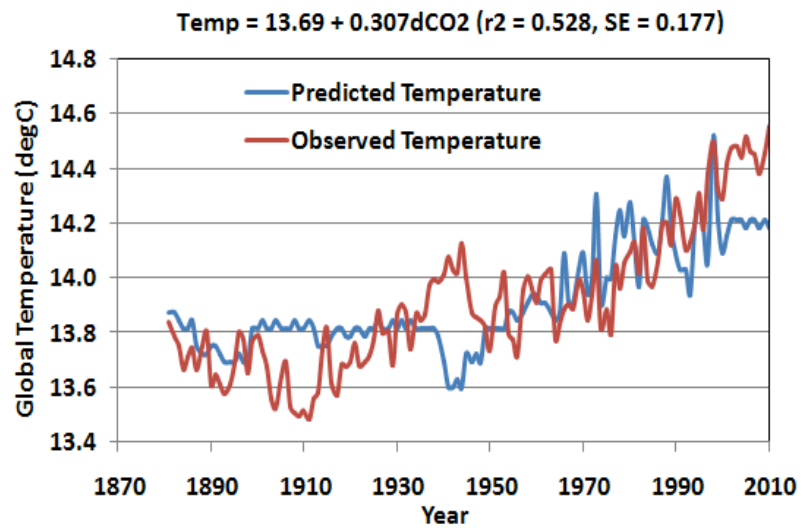

Figure 10: Regression analysis for global temperature versus $\mathrm{CO}_{2}$ (left) and global temperature versus annual increment of $\mathrm{CO}_{2}$ (right). 

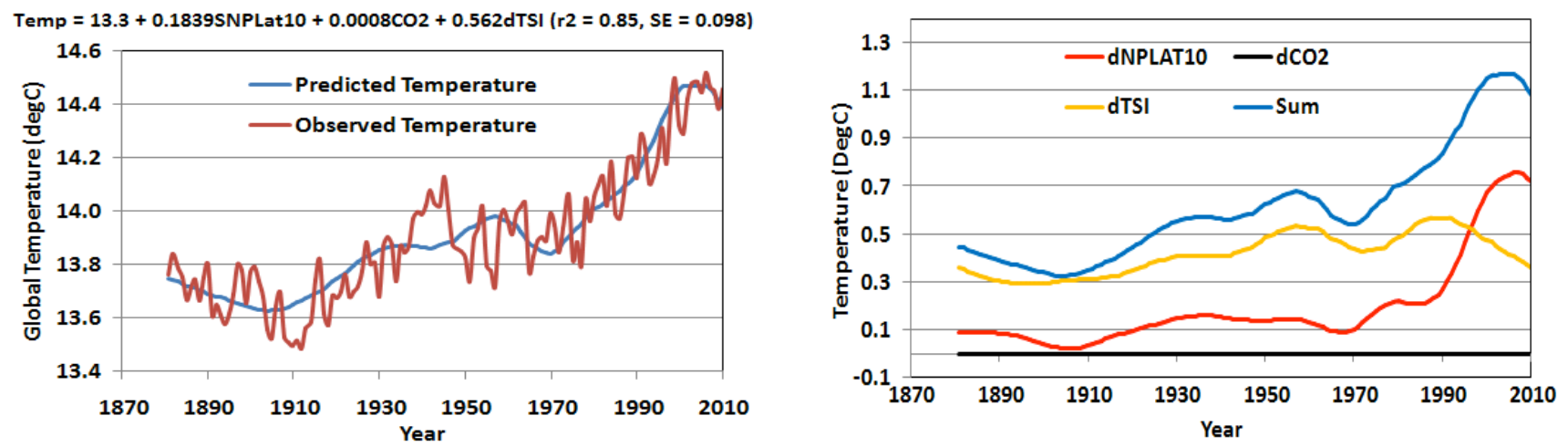

Figure 11: Global temperature anomaly versus decadal change of North Pole shift, annual increment of $\mathrm{CO}_{2}$, and TSI anomaly (left). Since the start of the new millennium, the relative contribution of North Pole shift is about $63.5 \%$, TSI is about $36.4 \%$, and $\mathrm{CO}_{2}$ change is about $0.1 \%$. The contributions of each climate predictor to global temperature are also shown (right).

insignificant contribution to global warming with about $0.1 \%$ contribution to the observed global temperature rise. This result falsifies the anthropogenic global warming theory as clearly shown by the big impact of both the North Pole tilt and TSI changes as compared to the insignificant contribution of annual $\mathrm{CO}_{2}$ change (Figure 11, right). The effect of polar shift outweighed the combined contribution of $\mathrm{TSI}$ and $\mathrm{CO}_{2}$ changes after 1995 when global temperatures exceeded existing records.

\subsection{Preliminary Global Climate Simulations}

The Global Climate Model-EdGCM simulates global processes on a coarse horizontal resolution of $8^{\circ} \times 10^{\circ}$ $x 9$ vertical layers in the atmosphere. However, the use of EdGCM in simulating climate change due to planetary-scale changes on earth (i.e. increased planetary obliquity) and solar changes can help elucidate the major mechanism of the present global climate problem. The large-scale climate determines the environment for microscale (1 $\mathrm{km}$ or less) and mesoscale (from several kilometers to several hundred kilometers) processes that govern weather and local climate [27]. Hence, even the EdGCM could be used to understand the impact of important physico-chemical processes on earth or basic mechanisms that are external in nature. For instance, changes in solar irradiance or the increase of destructive earthquakes and the concomitant tilting of the earth on its axis (i.e. enhanced obliquity) could now be simulated. The possible axial tilting of the earth as a result of the great Sumatra earthquake that triggered the Asian Tsunami in 2004, and the great Chile earthquake of February 2010 had been documented by the National Aeronautic and Space Administration (NASA). In addition, changes in the solar constant (luminosity) had also been observed using open solar magnetic field [25]. Slight changes in the solar output had also been detected by NASA using estimations of the solar meridional circulation and the associated magnitude of its velocity. The potential impact of enhanced obliquity on the global climate is evaluated in this modeling study while the solar output was assumed constant.

The average obliquity used by global climate models at present is about $23.44^{\circ}$ and is normally assumed constant throughout the simulation. This value however, can be varied in the climate model EdGCM. To get an appreciable impact on the earth's climate, the model assumes that the earth's tilt has been forced to increase from $23.4^{\circ}$ to about $28.4^{\circ}$, or an increase of $5^{\circ}$ from the average value. The improved astronomical model of Girkin showed that the maximum axial tilt of the earth could be higher than the Milankovitch-predicted obliquity [6]. A 1-million year simulation that included the gravitational interaction between the earth, moon, sun and the other planets of the solar system showed that the earth's obliquity may vary from $19-28.4^{\circ}$ about the ecliptic plane and not 21 $25^{\circ}$ as initially estimated by Milankovitch.

To determine the potential impact of planetary tilting on the global climate, the concentrations of various greenhouse gases (GHG) had been assumed constant in the EdGCM climate model. Constant GHG concentrations (fixed for 1970, 1988 and even during the pre-industrial era) had been assumed in the model simulations. This was done to isolate the impact of enhanced obliquity on the global climate.

Assuming that the planetary obliquity has been forced to a maximum value of $28.4^{\circ}$, the EdGCM climate model was run with constant $\mathrm{CO}_{2}$ and constant 
solar luminosity. The initial results of the model show that the global surface air temperature within the period from $1988-2008$ would increase from $13.4-14.2^{\circ} \mathrm{C}$. The global (and ocean) temperature increase during the first 10 years is calculated by the model to be more abrupt with about $0.6^{\circ} \mathrm{C}$ increase. The estimated warming in the succeeding 10 years is only about $0.18^{\circ} \mathrm{C}$. On land, a similar increasing but fluctuating trend is predicted except for a slightly lower magnitude of warming from $9.15-8.37^{\circ} \mathrm{C}$ or a total of $0.77^{\circ} \mathrm{C}$ increase for the 20-year period simulated. In addition, the simulated sea surface temperature (SST) has also increased from a global average value of $17.8-18.3^{\circ} \mathrm{C}$ or about $0.46^{\circ} \mathrm{C}$ increase. The steeper rise in the simulated temperature as compared to observed values is due largely to the complementing effect of the greenhouse gases which could not be zeroed during the simulations.

Although fluctuating, the climate model also simulated a general increase in global precipitation and evaporation. It should be noted that the simulated slight increase is averaged for the whole planet and that higher temperature rise and greater precipitation can occur regionally. Furthermore, the model simulated a decrease in ocean ice and snow cover. A decrease of ocean ice cover of about $3 \%$ has been simulated by the model for the period of 1988-2008. This is not far from observed reduction of ice sheet in the northern hemisphere (i.e. about $4 \%$ decrease in ice cover within the same period). Again, the predicted reduction of ice cover is averaged throughout the whole planet. The general decrease of snow cover due to melting of ice caps (and glaciers) and the associated exposure of organic soil in the polar regions could have facilitated the emission of a vast storage of carbon and methane. In addition, sinking icy water can pump out stored carbon dioxide beneath the cold ocean surface. Thus, the observed poleward increase in GHG concentrations and polar amplification of warming can be explained solely by the forced axial tilting.

The enhanced planetary obliquity and its profound effect on the reduced ice cover can now explain the global warming problem, with the poles warming at a faster rate than other regions on earth. Global temperature anomaly record from NCDC shows a $0.5^{\circ} \mathrm{C}$ increase during the period simulated. While the simulated increase of $0.7^{\circ} \mathrm{C}$ is slightly higher, this is largely due to overestimated effect of $\mathrm{CO}_{2}$ (via feedback on water vapor increase) since it cannot be zeroed. The simulated higher temperature as compared to the observed global temperature rise could only be explained by the forced axial tilt of the planet due to the concomitant increase in the absorbed solar radiative flux.

Even if $\mathrm{CO}_{2}$ is fixed at pre-industrial values, EdGCM model results showed that the absorbed global radiation and net heating increases due to axial tilting alone. The model result showed an increase in the solar radiative forcing of about $1.1 \mathrm{~W} / \mathrm{m}^{2}$ even if $\mathrm{CO}_{2}$ concentration did not increase since the pre-industrial period. It should be noted that the observed radiative forcing within a period of about 30 years (1975-2005) is about $0.6-1.7 \mathrm{~W} / \mathrm{m}^{2}$ and the modeled radiative forcing is just within this range.

\subsubsection{Comparison of Observed and Simulated Global Temperature}

Assuming that the planetary obliquity has been enhanced to a value of about $28.4^{\circ}$, EdGCM was run and the model result revealed a close approximation of the observed global temperature anomaly (Figure 12). Only the short period from 1988-2008 was simulated since abrupt obliquity change started to occur only from the 1990's when major earthquakes increased in

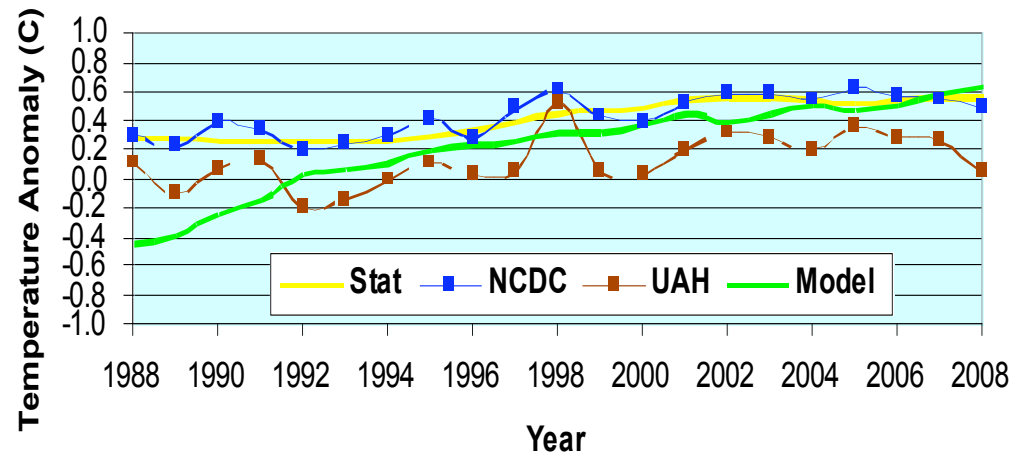

Figure 12: Observed and predicted global temperature anomaly $(C)$. The green line is averaged from the EdGCM model results with constant $\mathrm{CO}_{2}$ in $1988 \& 1978$. The model results are departure from a mean global temperature of $13.9^{\circ} \mathrm{C}$. 
frequency of occurrence. The UAH data set (satellitederived) was taken from the University of Alabama in Huntsville (www.vortex.nsstc.uah.edu/data) [28]. They represent global temperature anomalies in the lower troposphere and are therefore slightly lower than the NCDC temperature anomalies obtained from surface stations on land and over the oceans.

From 1992 onwards, the model result showed close agreement between the observed and simulated global temperature anomaly. This corresponds to the period when earthquake count (and planetary obliquity) started to increase rapidly. The small discrepancy between the observed and simulated values before this period could be attributed to the additional GHG impact as the EdGCM model could not be run without the GHG forcing (e.g. zero GHG concentrations did not work). The slightly steeper increase in the simulated global temperature could be attributed to the slightly higher obliquity assumed in the model as compared to the actual value. It should be noted that the observed obliquity value is now about $27.5^{\circ}$. This value could not be varied annually in the model and was increased immediately from the start of simulation.

The model results using the EdGCM also showed realistic increases of sea surface temperatures (SST), evaporation and precipitation, while snow cover was shown to decrease when the obliquity is increased. It was shown by the climate model that even if $\mathrm{CO}_{2}$ and other gases do not increase, the absorbed global radiation and net heating of the planet increases due to axial tilting alone. Even if $\mathrm{CO}_{2}$ is fixed at pre-industrial values, the global radiation and heating of the planet was simulated to increase due to enhanced obliquity.

\section{CONCLUSIONS AND RECOMMENDATION}

Analysis of the observed number of major earthquakes, geomagnetic polar shift and estimated obliquity, total solar irradiance, global temperature, and greenhouse gases showed that the major factor affecting the global warming and climate change problem is obliquity change. This is consistent with Drysdale et al. which found that obliquity changes have caused previous global warming events [28]. The present study showed in several ways that the present global warming problem including GHG rise is due mainly to earthquake-perturbed obliquity change and changes in the total solar irradiance. First, the observed motion of the geomagnetic poles showed that the North Pole has abruptly shifted since 1970. Evidently, this has caused the temperature and $\mathrm{CO}_{2}$ concentrations to rise dramatically since then. The polar shift data showed that major earthquakes and associated forces and applied torques within the earthocean interface proved to be the major cause of enhanced planetary tilt as shown by the sudden obliquity change when the frequency of major earthquakes started to increase. Second, the obliquity change was computed from the perturbed lunar force of attraction during the occurrence of great earthquakes (e.g. giant Sumatra quake and tsunami of December 2004. It was shown by the EPOCH model developed here, how the obliquity was enhanced by a strong oceanic pressure during the gian earthquake and tsunami in the Indian Ocean in December 2004. This also corroborated the hypothesis of Drysdale et al. that the current deglaciation might have started in the South Pole as it tilted towards the sun during the winter solstice when the North Pole tilted away from the sun [28]. Third, measurements of the solar positions at sunrise and sunset during the summer and winter solstices of 2010-2011 revealed that the obliquity of the earth has indeed increased to $27.5^{\circ}$. Fourth, the solar radiation model SOLRAD also revealed a net gain in solar radiative flux on earth due to enhanced obliquity. This simplified model clearly showed that the enhanced planetary tilt was the cause of the poleward amplification of climate change due to the poleward increase of net solar radiative flux absorbed by the planet. The estimated increase in the planetary tilt, which is within the newly computed obliquity of Girkin, was also shown by SOLRAD to be the cause of the observed net gain in the solar radiative flux of about $\mathbf{2 . 8}$ $\mathrm{W} / \mathrm{m}^{2}$ which has been wrongly attributed to greenhouse gases [6]. Finally, global climate simulations using EdGCM also showed that enhanced planetary obliquity could lead to increased atmospheric and sea surface temperatures, increased evaporation and precipitation, and decline of snow and ocean ice cover in agreement with global observations. Using constant GHG concentrations, the simulated increase in the global temperature was shown to agree well with observations from the NCDC. The observed polar amplification of climate change is a strong manifestation that seismicenhanced obliquity through oceanic pressure forces and the concomitant increase of absorbed solar radiation by higher latitudes, is the real cause of the global warming problem and GHG rise presently occurring on earth. As major earthquakes occur in the future, the obliquity could reach the maximum value of $28.4^{\circ}$ which would further increase the net radiative flux on earth resulting to faster decline of polar ice mass, more forest fires and GHG emission during the 
summer, and a considerable impact on the weather and climate on earth. The warming of the earth's surface itself due to increased solar radiative flux from enhanced obliquity could have started the increasing amount of high clouds and water vapor concentration in the atmosphere. The results of this study differ from Trenberth \& Fasullo which concluded that global warming from increasing absorbed solar radiation was due to decreasing cloud amount [29].

The climate simulations showed that when $\mathrm{CO}_{2}$ concentration is increased at present day values and held constant throughout the simulation, the surface temperature, evaporation and precipitation all increased. The climate model appears to yield results that show overestimated global warming due solely to $\mathrm{CO}_{2}$ and $\mathrm{GHG}$ increases. Decreasing the $\mathrm{CO}_{2}$ concentration to pre-industrial values resulted to unrealistic cooling and decreased evaporation and precipitation. It is therefore recommended that the atmospheric chemistry module on GHG be temporarily removed from existing Global Climate Models and could be run with variable obliquity. It is further recommended that more observational studies be conducted to determine the change in planetary obliquity. More studies should also be conducted to determine the relationship between GHG emission and seismic disturbances as the data analysis suggested that even $\mathrm{CO}_{2}$ increase was significantly correlated with the North Pole shift and obliquity changes.

\section{ACKNOWLEDGMENT}

This study was fully funded by Hymetocean Peers Co., Antipolo City, Philippines. The tilting of the earth on its axis by the Great Sumatra Quake and Tsunami of December 2004 was revealed in the $7^{\text {th }}$ Secret of Emmitsburg (www.centeroftheimmaculateheart.org). Meaningful discussions concerning the global warming and climate change problem with Professor Mariano A. Estoque (1997 IMO Awardee) of the Manila Observatory is gratefully acknowledged.

\section{REFERENCES}

[1] Croll J. On the change in the obliquity of the ecliptic: It's influence on the climate of the polar regions and level of the sea. Trans Geol Soc 1867; Glasgow.

[2] Intergovernmental Panel on Climate Change (IPCC). Climate change 2007: The physical science basis. Contribution of working group I to the fourth assessment report (FAR) of the intergovernmental panel on climate change. Solomon S, Qin $D$, Manning $M$, et al., Eds, Cambridge University Press, Cambridge 2007.

[3] Jaworowski Z. CO2 scandal. 2007; Spring/Summer $21^{\text {st }}$ Century Science and Technology.
Bond-Lamberty B, Thomson A. Temperature-associated increases in the global soil respiration record, Nature 2010. doi:10.1038/nature08930. http://dx.doi.org/10.1038/nature08930

[5] Meehl GA, Washington WM, Ammann CP, Arblaster JM, Wigley TML, Tehaldi C. Combinations of Natural and Anthropogenic Forcings in Twentieth-Century Climate. J Climate 2004; 17: 3721-7.

http://dx.doi.org/10.1175/1520-

0442(2004)017<3721:CONAAF>2.0.CO;2

[6] Girkin AN. A computational study on the evolution of the dynamics of the obliquity of the earth. Master of Science Thesis, 2005; Miami University.

[7] Laskar J, Joutel F, Robutel P. Stabilization of the Earth's obliquity by the Moon. Nature 1993; 361: 615-7. http://dx.doi.org/10.1038/361615a0

[8] http://www.jpl.nasa.gov/releases/2000/cha-ndlerwobble.html. A Mystery of Earth's Wobble Solved: It's the Ocean.

[9] Rivera PC. Modeling the Asian tsunami evolution and propagation with a new generation mechanism and a nonlinear dispersive wave model. Sci Tsunami Haz 2006; 25: 1833.

[10] http://edgcm.columbia.edu. The educational Global Climate Model (EdGCM Ver 3.2). Columbia University. New York.

[11] http://en.wikipedia.org/wiki/List_of_21st_century_earthquake s\#cite_note-34

[12] http://en.wikipedia.org/wiki/List_of_20th_century_earthquake s\#cite_note-34

[13] http://www.ncdc.noaa.gov

[14] http://www.ngdc.noaa.gov/geomag/data/poles/NP.xy

[15] Chao BF, Iz HB. Does polar motion influence the occurrence of earthquakes. EOS, Trans Amer Geophys Union 1992; 73: 79.

[16] Rivera PC. Modeling the mysterious tsunami swirls off the northeast coast of Japan during the great Tohoku earthquake of March 2011. Submitted to Sci. Tsunami Hazard 2011.

[17] Song YT. Detecting tsunami genesis and scales directly from coastal GPS stations. Geophys Res Lett 2007; 34: L19602. doi:10.1029/2007GL031681. http://dx.doi.org/10.1029/2007GL031681

[18] Song Y, Fu L, Zlotnicki V, Ji C, Hjorleifsdottir V, Shum C, Yi $Y$. Horizontal motions of faulting dictate the 26 December 2004 Tsunami Genesis. American Geophysical Union (AGU) Fall Meeting Dec. 2006, abstract \#U53C-02

[19] Humlum O, Solheim JE, Stordahl K. Identifying natural contributions to late Holocene climate change. Global and Planetary Change 2011; 79: 145-56.

[20] Kosek W, McCarthy DD, Luzum BJ. Variations of annual oscillation parameters, El Niño and their influence on polar motion prediction errors. Accepted in Studia Geophysica et Geodetica Warsaw, Poland, 2001.

[21] Pelletier G. SOLRAD Version 1.2. A solar position and radiation calculator for Microsoft Excel/VBA. Washington State Department of Ecology, Olympia, WA. Bird RE, Hulstrom RL (1991) A Simplified Clear Sky model for Direct and Diffuse Insolation on Horizontal Surfaces. SERI Technical Report SERI/TR-642-761, Feb 1991. Solar Energy Research Institute, Golden, CO, 1995.

[22] Bras RL. Hydrology. Addison-Wesley, Reading, MA, 1990.

[23] Ryan PJ, Stolzenbach KD. Engineering aspects of heat disposal from power generation, (D.R.F. Harleman, ed.). R.M. Parson Laboratory for Water Resources and Hydrodynamics, Department of Civil Engineering, Massachusetts Institute of Technology, Cambridge, MA, 1972.

[24] Steinhilber F, Beer J, Fröhlich C. Total solar irradiance during the Holocene. Geophys Res Lett 2009; 36: L19704. 
doi:10.1029/2009GL040142.

http://dx.doi.org/10.1029/2009GL040142

[25] Fang JY, Zhu JL, Wang SP, Yue C, Shen HH. Global warming, human-induced carbon emissions, and their uncertainties. Sci Chin Earth Sci 2011; 54: 1458-68. http://dx.doi.org/10.1007/s11430-011-4292-0

[26] Hurrell J, Meehl GA, Bader D, Delworth TL, Kirtman B, Wielicki B. A Unified Modeling Approach to Climate System Prediction. BAMS 2009; 90: 1819-32. doi:10.1175/2009BAMS2752.1. http://dx.doi.org/10.1175/2009BAMS2752.1

[27] http://vortex.nsstc.uah.edu/data/msu/t2lt/

[28] Drysdale RN, Hellstrom JC, Zanchetta G, Fallick AE, Sánchez Goñi MF, Couchoud I, McDonald J, Maas R,
Lohmann G and Isola I. Evidence for obliquity forcing of glacial termination II. Published Online Aug. 13, 2009. Science 18 Sept. 2009; 325: 1527-31. DOI: 10.1126/science. 1170371.

http://dx.doi.org/10.1126/science.1170371

[29] Trenberth KE. Fasullo JT. Global warming due to increasing absorbed solar radiation. Geophys Res Lett 2009; 36: L07706. doi:10.1029/2009GL037527. http://dx.doi.org/10.1029/2009GL037527

[30] Fujita M, Chao BF, Sanchez BV, Johnson TJ. Oceanic torques on solid Earth and their effects on Earth rotation. J Geophys Res 2002; Vol. 107: 2154, 13 pp. doi:10.1029/2001JB000339

(C) 2012 Rivera et al.; Licensee Lifescience Global.

This is an open access article licensed under the terms of the Creative Commons Attribution Non-Commercial License (http://creativecommons.org/licenses/by-nc/3.0/) which permits unrestricted, non-commercial use, distribution and reproduction in any medium, provided the work is properly cited. 